\title{
Forecasting of Sporadic Demand Patterns with Seasonality and Trend Components: An Empirical Comparison between Holt-Winters and (S)ARIMA Methods
}

\author{
Rita Gamberini, ${ }^{1}$ Francesco Lolli, ${ }^{1}$ Bianca Rimini, ${ }^{1}$ \\ and Fabio Sgarbossa ${ }^{2}$ \\ ${ }^{1}$ Department of Engineering Sciences and Methods, University of Modena and Reggio Emilia, \\ via Amendola 2, Padiglione Morselli, Reggio Emilia 42100, Italy \\ 2 Department of Management and Engineering, University of Padua, Stradella San Nicola 3, \\ Vicenza 36100, Italy
}

Correspondence should be addressed to Rita Gamberini, rita.gamberini@unimore.it

Received 19 March 2010; Accepted 16 June 2010

Academic Editor: Carlo Cattani

Copyright (C) 2010 Rita Gamberini et al. This is an open access article distributed under the Creative Commons Attribution License, which permits unrestricted use, distribution, and reproduction in any medium, provided the original work is properly cited.

\begin{abstract}
Items with irregular and sporadic demand profiles are frequently tackled by companies, given the necessity of proposing wider and wider mix, along with characteristics of specific market fields (i.e., when spare parts are manufactured and sold). Furthermore, a new company entering into the market is featured by irregular customers' orders. Hence, consistent efforts are spent with the aim of correctly forecasting and managing irregular and sporadic products demand. In this paper, the problem of correctly forecasting customers' orders is analyzed by empirically comparing existing forecasting techniques. The case of items with irregular demand profiles, coupled with seasonality and trend components, is investigated. Specifically, forecasting methods (i.e., HoltWinters approach and (S)ARIMA) available for items with seasonality and trend components are empirically analyzed and tested in the case of data coming from the industrial field and characterized by intermittence. Hence, in the conclusions section, well-performing approaches are addressed.
\end{abstract}

\section{Introduction}

In the recent competitive environment, where manufacturing and service companies operate in unstable sectors, managing irregular and sporadic demand patterns represents an increasingly frequent and complex issue. Startup productions, multiechelon supply chains or spare parts production, and selling are some examples of market fields characterized by intermittent demand profiles. 
The complexity of dealing with these kinds of demand patterns lies in finding the best tradeoff between negative effects related with high storage levels, such as high amount of space and resources for keeping large warehouse areas, high holding costs, as well as high risks and cost due to items obsolescence, and negative effects related with low storage levels, such as lost demand and customers.

Therefore, when treating irregular and sporadic demand patterns, two relevant issues are discussed:

(i) demand forecasting in the future periods,

(ii) utilization of demand forecasting obtained for managing stocks. Hence issues related with when and how much it costs to create stocks for satisfying the forecasted customers' orders are faced.

The focus of this paper is on the first issue, which represents an unforgettable prerequisite for the second one and could become a needful competitive leverage for companies.

\section{Literature Review}

Croston [1] has published a pioneer work concerning forecast of irregular and sporadic demand (successively improved by Rao [2]). He observes that single exponential smoothing, even if frequently used for forecasting in inventory control systems, reaches inappropriate results when applied to intermittent demand patterns. Otherwise, computing both the average size of not-null demand occurrences and the average intervals between such occurrences is the intuition of Croston in order to achieve the estimator of mean demand per period. In particular, Croston considers customers' order series with demand occurrences generated by a Bernoulli process and with demand sizes (when not null) following a normal distribution. Then, he applies separately a single exponential smoothing to not-null demand sizes and interdemand intervals. Finally he combines them.

Successively, modifications of Croston's approach are proposed. Johnston and Boylan $[3,4]$ analyse demand patterns with the order arrival process modelled as a Poisson stream. Therefore, a negative exponential distribution is supposed to represent interorder arrivals. The authors propose a model to estimate the variance of demand and use it in a forecasting demand approach, whose performance is tested by considering a wide variety of operative conditions (i.e., many different average interdemand intervals, negative exponential, Erlang, and rectangular as distributions of order size). Syntetos and Boylan [5] explain the detection of a mistake in Croston's mathematical derivation of the expected estimate of demand per time period and propose an alternative approach, based again on the concept of forecasting demand from its constituent events. Subsequently, Syntetos and Boylan [6] introduce a factor equal to $(1-\alpha / 2)$ applied to Croston's original estimator of mean demand, with $\alpha$ being equal to the smoothing parameter in use for updating the interdemand intervals, in order to obtain a theoretically unbiased estimator. The derivation of the new estimator is based on Croston's assumptions of stationary, identically, independently distributed series of demand sizes and demand intervals, geometrically distributed interdemand intervals, and independence of demand sizes and intervals. Segersted [7] has proposed an alternative Croston's approach modification, adopted in sporadic demand inventory control by coupling it to the computation of probability of stock shortage, supposing demand following an Erlang distribution. An experimental analysis of the reachable performance is reported by Levén and Segersted in [8]. 
Recently, original contributions are published. Willemain et al. [9] forecast the cumulative distribution of demand over a fixed lead time using a new type of time series bootstrap. Specifically, the hypothesis of demand independence among subsequent time periods is disregarded and the existence of autocorrelation is considered. In the study by Gutierrez et al. in [10] the problem of forecasting lumpy demand series is analysed and a Neural Network (NN) approach is proposed. NN outperforms single exponential smoothing, Croston's approach, as well as Syntetos and Boylan approach in most of the analysed series and forecasting environments. Nevertheless, a consistent amount of data is required for setting the estimator and this is not the case treated in what follows in the paper. Keeping our attention on methodologies requiring a low amount of data to be set (i.e., moving averages, exponential smoothing, Croston's approach, Syntetos and Boylan modifications, as well as time series bootstrap), several works publish results obtained by comparing their performance in a wide variety of alternative operative conditions (i.e., Willemain et al. [11], Johnston and Boylan [3, 4], Sani and Kingsman [12], Strijbosch et al. [13], Willemain et al. [9], Syntetos and Boylan [6], Regattieri et al. [14], and given specifications reported by Syntetos in [15]).

Since Syntetos et al. [16] published their work, experimental analyses on alternative forecasting methods have been carried out by following the steps described in the sequel: firstly data categorization based on characteristics chosen by the management in respect to its needs and then finding the more appropriate forecasting methods for the different categories. Otherwise, Syntetos et al. [16] propose to categorize the demand patterns by following an alternative procedure: firstly analysing the optimal performance areas of several forecasting methods and then categorizing the demand patterns in accordance with results obtained. Even if, on one hand, these pioneering passes in the direction of demand patterns' categorizing are very interesting, on the other hand, the study by Syntetos et al. in [16] is based on assumptions not always confirmed by real-life data (i.e., demand occurring as a Bernoulli process or independence of demand values, as commented by [17-19]). Moreover, the proposed categorization methodology, even if it achieves satisfying results in the applied statistical fitting tests, does not describe the behaviour of the whole of the data series introduced in the experimentation. Hence, when complex data coming from reallife industrial contexts are managed, the definition of a pool of well-performing forecasting techniques still remains an interesting result to be investigated. Specifically, in this paper sporadic and irregular demand patterns with seasonality and trend components are studied.

Forecasting methods for demand patterns with seasonality and trend components are proposed by several authors. The focus in the following brief overview is on two techniques: the Holt-Winters (HW) approach [20], (see [21]), and ARIMA model, which is identified and then applied through the Box-Jenkins procedure [22]. Such a choice is justified by their applicability in real-life environments, mainly due to the great multitude of available statistical commercial softwares and expected good results, justified by past studies cited in the sequel. Furthermore, whilst HW is a useful forecasting tool addressed for its simplicity, (S)ARIMA is a robust approach appreciable due to its applicability to a wide variety of operative conditions.

HW is an extrapolative technique that isolates level, trend, and seasonal components of a time series regardless of the nature of the time series data being collected. It presents both a multiplicative and an additive version. ARIMA model is an integrated technique of auto-regressive (AR) models and moving average models, capable of finding a fitting function in an iterative way through the Box-Jenkins procedure. In the following, the acronym (S)ARIMA is used in place of ARIMA to specify the possibility that seasonality is present in 
analysed time series. For a more detailed discussion on the application of (S)ARIMA models see the studies by Jarrett in [23] and Bowerman and O'Connell in [24].

Several authors investigate HW and (S)ARIMA performances in a wide variety of operating conditions [25-28], and [29]. Nevertheless the analysis of reachable forecasting results on irregular and sporadic time series with trend and seasonal components still remains a field to be widely investigated.

Hence, the purpose of the paper is to present results obtained by comparing HW and (S)ARIMA forecasting performances when applied to a set of real-life sporadic and irregular time series with seasonality and trend components.

The paper is organized as described in the sequel. A synthesis of the methodology implemented in the experimental analysis and then the first step of the project, concerning the collection and preliminary analysis of data, are presented, respectively, in Sections 3 and 4. In Section 5 the selection of the best (S)ARIMA model is carried out and then compared in Section 6 with the Holt-Winters method in terms of forecasting performances. Finally, conclusions and some guidance for practitioners are given in Section 7.

\section{Framework of the Experimental Analysis}

As aforementioned, the aim of the paper is the comparison between the Holt-Winters exponential smoothing with (S)ARIMA in cases of erratic and sporadic demands with seasonal and trend components.

Holt-Winters method manages three components of demand per period: a level component, a trend component, and a seasonal component. Each of them is estimated by exponential smoothing and successively opportunely weighted and combined in order to predict demand. In particular, two versions of HW components compositions are available: additive and multiplicative, but the presence of time periods with null demand does not allow the multiplicative version to be applied in this paper [20] (Winters, 1960). A linear regression on time values is used to define the initial level and trend components while a dummy-variable regression on detrended time values is used to estimate the initial seasonal component.

While HW is simply applied by commercial softwares (in the sequel EViews 5 is adopted), which allow the solution to be achieved without any intervention of the user, (S)ARIMA models require the optimal definition of a set of parameters in accordance with results obtained in fitting tests. In Figure 1 the Box-Jenkins procedure [22] is briefly depicted and then explained in the sequel.

The flow diagram depicted in Figure 1 is an iterative decisional framework finalized firstly to find a (S)ARIMA model and then to apply it for demand forecasting. The BoxJenkins procedure starts from the collection of data and preliminary analysis, which allow the identification of the preferable (S)ARIMA model to be achieved quickly. The first step of the preliminary analysis regards the stationarity of the time series, in terms of both mean and variance, required as a prerequisite for the application of the auto-regressive (AR) models and moving average (MA) models [22]. In case of nonstationarity, the procedure suggests the introduction of two differentiation orders, that is, $d$ and $D$, that are, respectively, the nonseasonal and seasonal differentiation orders. A first order $d$ of differentiation is applied in case of linear trend while a second order makes the time series stationary in case of quadratic trends and so on. In the same way $D$ regards the seasonal component and it can be chosen by several tests, such as the Canova and Hansen test [30]. 


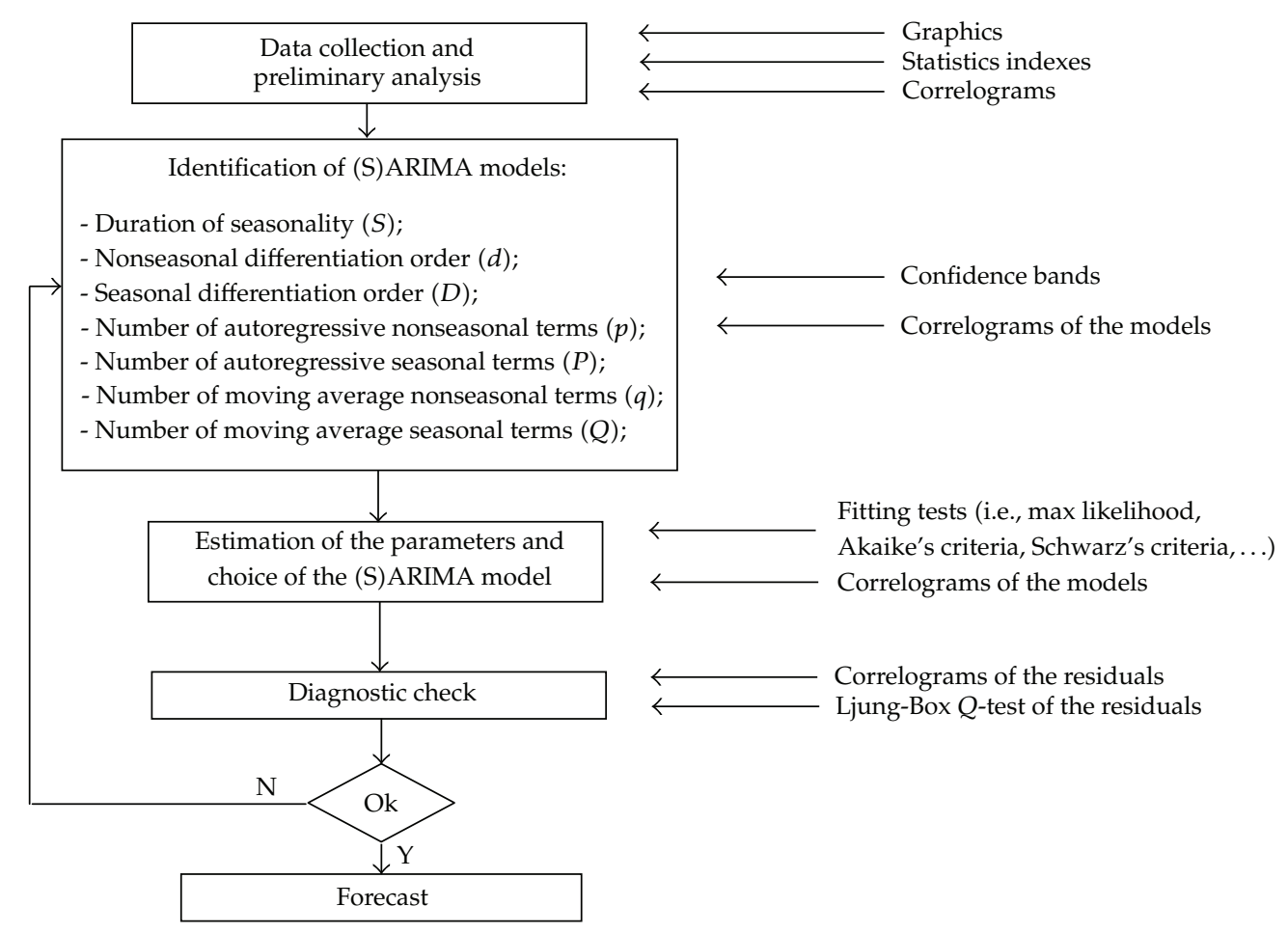

Figure 1: The Box-Jenkins procedure.

Graphics, statistical indexes, and correlograms support this phase. In particular, the more useful indicators are the distribution of the global autocorrelation coefficients $\left(\rho_{k}\right.$, for $k=1, \ldots, T$ with $T$ being equal to the number of time periods constituting the time series) and the distribution of the partial correlation coefficients, which emerge, respectively, from the analysis of the global correlogram (ACF) and the partial correlogram (PACF). When a time series is stationary, autocorrelation coefficients in ACF and PACF tend to zero after two or three time lags. Some tests are available in order to check the stationarity of the series, such as the Durbin-Watson test and the Ljung-Box $Q$-test. Moreover, ACF and PACF provide guidance both in extrapolating trend and seasonal components and then in selecting parameters $(S, p, q, P, Q)$ for the complete (S)ARIMA specification in respect of the adherence to theoretical models. In synthesis, the seven parameters $(S, d, D, p, q, P, Q)$ uniquely define each (S)ARIMA model that is suitable for fitting the original time series.

In order to chose the best (S)ARIMA model avoiding overfitting occurrence (the necessity of testing too many parameters), many techniques and methods have been suggested to add mathematical rigor to the search process, including Akaike's criterion [31] or Schwarz's criterion [32]. Each of them works by penalizing models based on the number of their parameters. Anyway, since nowadays statical commercial softwares allow the user to test different (S)ARIMA models very quickly, in the present paper the choice of the best (S)ARIMA model is based on its forecasting performances uniquely. Moreover, the identification of the best (S)ARIMA model throughout the whole application of the BoxJenkins procedure for decades has required specific statistical knowledge, finalized to define the seven (S)ARIMA parameters by graphics, statistical indexes, and correlograms analysis, 
which in real industrial implementations are available rarely. Nevertheless, nowadays, the purpose in the market of commercial softwares quickly testing different (S)ARIMA models guarantees the introduction of such procedure in a wide variety of real-life contexts.

After the identification of the (S)ARIMA model, a diagnostic check must be conducted (see Figure 1) for assessing that the model does not neglect any component. Thus, if residuals are correlated, then the Box-Jenkins procedure restarts from the (S)ARIMA model identification until the residuals are uncorrelated and normally distributed. The Ljung-Box $Q$ test helps the user to check the uncorrelation of the residuals. Finally, after a positive response given by the diagnostic check, demand forecast via (S)ARIMA can be made.

Several accuracy measures are presented in literature for comparing the performances of forecasting methods. For a more detailed discussion about them, see the study by Makridakis in [33].

Define $T$ as the number of forecasted time periods, $F_{t}$ as the forecasted demand size in time period $t, A$ as the mean demand size occurring in the forecasted time periods, and $D_{t}$ as the real demand size occurring in time period $t$, for $t=1, \ldots, T$.

Accuracy measures adopted in this paper are described in (3.1), (3.2), and (3.3), in accordance with guidelines reported by Regattieri et al. In [14].

\section{$M A D / A$}

It represents the Mean Absolute Deviation (MAD) divided by the average demand size. This index, by describing the incidence of the mean absolute forecasting error on the mean existing demand, allows the evaluation of forecasting approaches performance on time series with very different mean values, as introduced by Regattieri et al. [14]:

$$
\mathrm{MAD} / A=\frac{\sum_{t=1}^{T}\left|F_{t}-D_{t}\right| / T}{A}
$$

$M S E / A$

It represents the arithmetic Mean of the Sum of the Squares of the forecasting Errors (MSE) divided by the average demand size. Low values of MSE/ $A$ address the adoption of forecasting approaches with a high incidence of low errors between true values and estimated ones. Otherwise, high MSE/ $A$ indicates that high errors sometimes occur. Specifically, the ratio with $A$ is proposed again in order to compare values obtained in series characterized by consistent differences in the mean demand size:

$$
\mathrm{MSE} / A=\frac{\sum_{t=1}^{T}\left(F_{t}-D_{t}\right)^{2} / T}{A} .
$$

$M E / A$

It represents the Mean Error (ME) divided by the average demand size. This index permits to define the estimation behavior of forecasting methods and specifically to understand whether an overestimation or an underestimation of the prediction data occurs:

$$
\mathrm{ME} / A=\frac{\sum_{t=1}^{T}\left(F_{t}-D_{t}\right) / T}{A} .
$$


Specifically, in this proposed paper, the goodness of forecasting is evaluated by computing $\mathrm{MAD} / A, \mathrm{ME} / A$ and MSE/ $A$ on 5 and 12 future time periods.

\section{Collection and Preliminary Analysis of Data}

Twelve data series describing demand of twelve spare parts have been collected from real industrial applications, each of them composed by 36 time periods. In detail, the data are related to several high-value minuteria products, like precision screws and small spare parts for transmission and hydraulic units. They are all characterized by erratic patterns because of their variability in demand sizes while some data series are sporadic too due to the presence of time periods in which demand does not occur. Therefore, two coefficients are computed (CV and ADI) in accordance with definitions reported by Willemain et al. In [11]. Specifically, CV represents the coefficient of variation of not-null demands, while ADI represents the average number of time periods between two successive not-null demands. Alternatively, in accordance with definitions reported by Syntetos and Boylan in [5], $\mathrm{CV}^{2}$ can be computed, that is, the squared version of CV. Hence CV and ADI establish the marks, respectively, of demand sizes variability and of the intermittence of demand pattern.

In the following sections forecast will concern five and twelve periods ahead; thus $\mathrm{CV}$ and ADI are calculated both for 31 time periods, from period 1 to period $31\left(\mathrm{CV}_{31}\right.$, $\left.\mathrm{ADI}_{31}\right)$ and for 24 time periods $\left(\mathrm{CV}_{24}, \mathrm{ADI}_{24}\right)$, from period 1 to period 24 . Respectively, 5 and 12 disregarded data are adopted as benchmark for testing the forecasting performance of the analyzed approaches (HW and (S)ARIMA). Moreover, the statistical analysis leads to deny the aprioristic assumption of Croston [1]; in fact demand sizes (when demand occurs) are not normally distributed and they are not mutually independent due to the presence of autocorrelation, as outlined below.

The analysis based on the Box-Jenkins procedure (see Figure 1) begins by investigating the more useful characteristics of time series for the declared purposes, such as firstly the extrapolation both of a trend and of a seasonal and then the identification of an adequate (S)ARIMA model capable of fitting the series. These characteristics are related with the distribution of the global autocorrelation coefficients $\left(\rho_{k}\right)$ and the distribution of the partial correlation coefficients, which emerge, respectively, from the analysis of the global correlogram (ACF) and the partial correlogram (PACF). All demand patterns are generated by nonstationary processes, since their autocorrelation coefficients in ACF and PACF do not tend to zero after two or three time lags. Hence, parameters $d$ and $D$ of the (S)ARIMA models must be achieved for each time series in order to make them stationary. Moreover, some series are mainly influenced by seasonality while others present both seasonality and a consistent trend. In fact, in both cases $\rho_{k}$ are different to zero or present peeks every $S$ time lag. But, in the former case they are characterized by disregardable increases or decreases by varying time lag, while in the latter case, when both seasonality and a consistent trend are registered, increases or decreases clearly appear.

Table 1 reports the summary of the main characteristics for each time series: CV, ADI, the best distribution functions that are not rejected in fitting demand sizes (ddp), and finally the presence of both seasonality and consistent trend components.

The software AutoFit has been used. It evaluates all the best fitting distribution functions in descending order of ranking. Sometimes it does not find any fitting function. Such cases are traced in column ddp of Table 1, by indicating the label reject. 
Table 1: Data collection and preliminary analysis.

\begin{tabular}{|c|c|c|c|c|c|c|c|c|}
\hline Series & Group & $\mathrm{CV}_{31}$ & $\mathrm{ADI}_{31}$ & $\mathrm{CV}_{24}$ & $\mathrm{ADI}_{24}$ & $\mathrm{ddp}$ & Seasonality & Trend \\
\hline s1 & & 1.80 & 1.35 & 1.79 & 1.26 & reject & $\mathrm{x}$ & \\
\hline s2 & & 1.54 & 1.11 & 1.45 & 1.09 & Geometric $(6.34 e-002)$ & $x$ & \\
\hline s3 & & 1.30 & 1.19 & 1.35 & 1.09 & Neg. Binomial $(1,4.75 e-002)$ & $x$ & \\
\hline s4 & 1 & 1.09 & 1.48 & 1.01 & 1.33 & Neg. Binomial $(3,7.76 e-002)$ & $\mathrm{x}$ & \\
\hline s5 & & 1.22 & 1.19 & 1.21 & 1.14 & Neg. Binomial $(2,4.11 e-002)$ & $\mathrm{x}$ & \\
\hline s6 & & 1.25 & 1 & 1.30 & 1 & reject & $\mathrm{x}$ & \\
\hline s7 & & 2.40 & 1.29 & 2.36 & 1.33 & reject & $\mathrm{x}$ & \\
\hline s8 & & 1.11 & 1.41 & 1.33 & 1.50 & reject & $\mathrm{x}$ & $\mathrm{x}$ \\
\hline s9 & & 2.38 & 1.35 & 1.77 & 1.14 & Neg. Binomial $(2,4.88 e-002)$ & $\mathrm{x}$ & $\mathrm{x}$ \\
\hline s10 & 2 & 1.63 & 1.41 & 1.69 & 1.41 & Neg. Binomial $(1,9.88 e-002)$ & $x$ & $\mathrm{x}$ \\
\hline s11 & & 1.28 & 1 & 1.42 & 1 & Neg. Binomial $(1,4.7 e-002)$ & $\mathrm{x}$ & $\mathrm{x}$ \\
\hline s12 & & 1.30 & 1 & 1.34 & 1 & Neg. Binomial $(2,1.6 e-002)$ & $x$ & $x$ \\
\hline
\end{tabular}

Time series are grouped into two sets: Group 1 and Group 2. The former includes series from s1 to s7, mainly characterized by seasonal component, while the latter includes series from $\mathrm{s} 8$ to $\mathrm{s} 12$, with both seasonal and consistent trend components.

\section{Identification of Possible (S)ARIMA Models and Choice of the Best One}

The implementation of the Holt-Winters method does not require any discretional intervention of the user because the commercial software adopted in this paper finds the best smoothing parameters in an iterative way. For this reason, the main portion of this section is focused on (S)ARIMA models identification.

In order to reduce the number of tested (S)ARIMA models, the differentiation orders $(d$ and $D)$ are initially set and kept unmodified in the following steps. They represent, respectively, the non seasonal and the seasonal differentiation orders finalized to make the series stationary. By analyzing the patterns, on one hand, only linear trends emerge; therefore a first order of non seasonal differentiation is necessary $(d=1)$. On the other hand, $D$ could be chosen through several tests, such as the Canova and Hansen test [30], but in the proposed experimentation a stable seasonal pattern is always present; thus the seasonality is effectively handled by stationary seasonal AR and MA terms $(D=0)$. Moreover the duration of seasonality of each time series is 4 time periods; hence $S$ is set to 4 .

Since a (S)ARIMA model is uniquely defined by seven parameters $(p, d, q) \times(P, D, Q)_{S}$, the number of possible (S)ARIMA models to be tested is reduced for the assumptions above in the following way: $(p, 1, q) \times(P, 0, Q)_{4}$, where $p, q, P$, and $Q$ change from one to three.

Note that each (S)ARIMA model could generate negative forecasted values, which are practically inconsistent. Thus, a null demand is imposed every time a negative value is forecasted.

In order to compare the different (S)ARIMA models, their forecasting performance is evaluated in terms of MAD/ $A$, as underlined in Section 3. In particular, the selection of each (S)ARIMA model is based on the minimization of MAD/ $A$. The selected models for forecasting both 5 and 12 time periods ahead are reported in Table 2. 
Table 2: Selected (S)ARIMA models for 5 and 12 time periods ahead.

\begin{tabular}{|c|c|c|c|}
\hline Series & Group & 5 time periods ahead & 12 time periods ahead \\
\hline s1 & & $(3,1,1) \times(2,0,2)_{4}$ & $(2,1,3) \times(2,0,2)_{4}$ \\
\hline s2 & & $(2,1,2) \times(3,0,1)_{4}$ & $(1,1,3) \times(2,0,2)_{4}$ \\
\hline s3 & & $(3,1,1) \times(3,0,2)_{4}$ & $(3,1,2) \times(2,0,2)_{4}$ \\
\hline s4 & 1 & $(1,1,2) \times(2,0,3)_{4}$ & $(1,1,3) \times(2,0,2)_{4}$ \\
\hline s5 & & $(2,1,2) \times(2,0,2)_{4}$ & $(3,1,2) \times(2,0,1)_{4}$ \\
\hline s6 & & $(1,1,2) \times(2,0,2)_{4}$ & $(3,1,2) \times(2,0,1)_{4}$ \\
\hline s7 & & $(2,1,2) \times(3,0,1)_{4}$ & $(1,1,1) \times(3,0,1)_{4}$ \\
\hline s8 & & $(3,1,2) \times(2,0,2)_{4}$ & $(1,1,1) \times(3,0,1)_{4}$ \\
\hline s9 & & $(2,1,2) \times(3,0,1)_{4}$ & $(2,1,2) \times(2,0,1)_{4}$ \\
\hline s10 & 2 & $(1,1,2) \times(3,0,1)_{4}$ & $(2,1,3) \times(2,0,1)_{4}$ \\
\hline s11 & & $(2,1,2) \times(3,0,3)_{4}$ & $(1,1,1) \times(2,0,2)_{4}$ \\
\hline s12 & & $(2,1,2) \times(2,0,1)_{4}$ & $(3,1,2) \times(2,0,1)_{4}$ \\
\hline
\end{tabular}

Table 3: Comparison between (S)ARIMA and HW based on MAD/ $A$.

\begin{tabular}{|c|c|c|c|c|c|}
\hline \multirow{2}{*}{ Series } & \multirow{2}{*}{ Group } & \multicolumn{2}{|c|}{ MAD/ $A 5$ time periods ahead } & \multicolumn{2}{|c|}{ MAD/ A 12 time periods ahead } \\
\hline & & (S)ARIMA & HW & (S)ARIMA & HW \\
\hline s1 & \multirow{7}{*}{1} & $4.9 \%$ & $4.9 \%$ & $9.3 \%$ & $8.9 \%$ \\
\hline s2 & & $9.7 \%$ & $14.2 \%$ & $13.8 \%$ & $13.8 \%$ \\
\hline s3 & & $13.2 \%$ & $11 \%$ & $33.5 \%$ & $21.5 \%$ \\
\hline s4 & & $7.3 \%$ & $6.9 \%$ & $8.9 \%$ & $21.1 \%$ \\
\hline s5 & & $2.3 \%$ & $4.8 \%$ & $4.7 \%$ & $7.6 \%$ \\
\hline s6 & & $1.4 \%$ & $1.8 \%$ & $4.1 \%$ & $2.8 \%$ \\
\hline s7 & & $1.4 \%$ & $0.2 \%$ & $5.3 \%$ & $5.5 \%$ \\
\hline s8 & \multirow{5}{*}{2} & $14.9 \%$ & $21.6 \%$ & $35 \%$ & $40.9 \%$ \\
\hline s9 & & $8.3 \%$ & $66.7 \%$ & $53.6 \%$ & $92.9 \%$ \\
\hline s10 & & $5.3 \%$ & $26.7 \%$ & $16.5 \%$ & $32.5 \%$ \\
\hline s11 & & $4.0 \%$ & $11.1 \%$ & $8.5 \%$ & $24.6 \%$ \\
\hline s12 & & $10.7 \%$ & $10.4 \%$ & $10.4 \%$ & $21.7 \%$ \\
\hline
\end{tabular}

Subsequently, selected (S)ARIMA models are also compared with those of HW in terms of MSE/ $A$ and ME/ $A$ in order to evaluate their capability of often generating low errors along with indicating their potential overestimation or underestimation.

\section{Experimental Analysis: Comparison Between (S)ARIMA and HW}

The results obtained by HW method are directly comparable with those achieved through selected (S)ARIMA model.

In Table 3 the comparison between HW model and selected (S)ARIMA model is carried out in terms of MAD/ $A$ for each time series. In particular, only the lower MAD/ $A$ achieved by the two methods are reported in percentage values.

In Figures 2 and 3, the achieved MAD/ $A$ values are plotted, respectively, for 5 and 12 time periods ahead, divided into groups defined before for series with seasonal component (Group 1) and for series with both seasonal and consistent trend components (Group 2). 


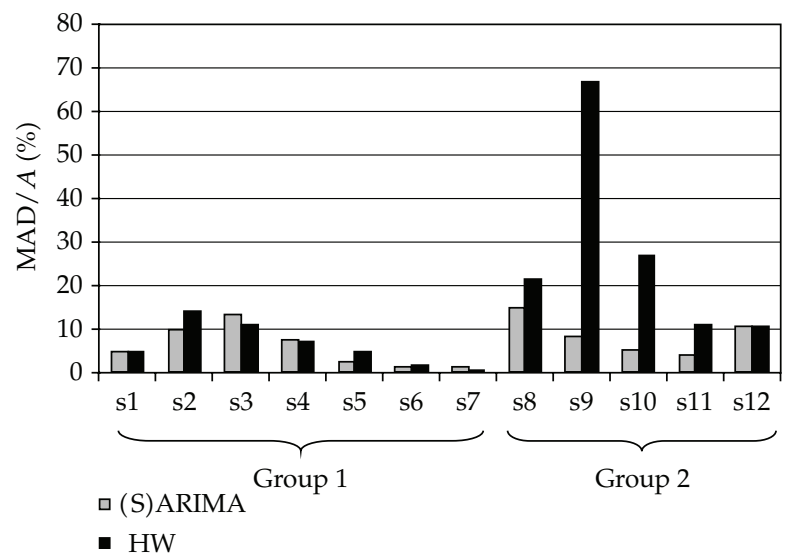

Figure 2: MAD/ $A$ values for each time series: forecasts on 5 time periods ahead.

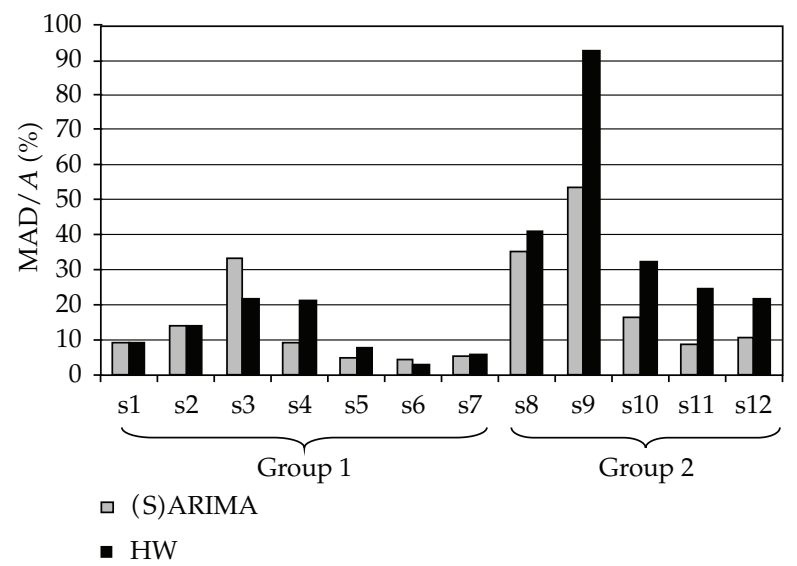

Figure 3: MAD/ $A$ values for each time series: forecasts on 12 time periods ahead.

In cases of forecasts on 5 time periods ahead and for time series belonging to Group 1 (from s1 to s7), the Holt-Winters method gives comparable results in respect of the best (S)ARIMA model found. In series s2, s5, s6, (S)ARIMA outperforms; in series s3, s4, s7, HW outperforms; in series s1, the same value of MAD/ $A$ is registered. Furthermore, the worst performing approach (HW for series s2, s5, s6 and (S)ARIMA for series s3, s4, s7) gives results not far from the best registered. Otherwise, when both seasonality and consistent trend occur (from s8 to s11), (S)ARIMA model guarantees performances better than those obtained by the Holt-Winters method. Time series s12 is the only case belonging to Group 2 in which Holt-Winters outperforms; however improvement induced is negligible.

Increasing the number of the forecasted time periods from 5 to 12 , the same guidelines can be traced. For time series belonging to Group 1, (S)ARIMA outperforms in s4, s5, s7, and HW outperforms in s1, s3, s6. The same MAD/ $A$ is reached in s2. Furthermore, except in the case of time series $s 3$, best MAD/ $A$ registered with HW and (S)ARIMA are comparable. Otherwise, in time series belonging to Group 2, (S)ARIMA outperforms and best MAD/ $A$ registered are consistent. In synthesis, the more complex the demand data series become, 
Table 4: Comparison between (S)ARIMA and HW based on MSE/ A.

\begin{tabular}{|c|c|c|c|c|c|}
\hline \multirow{2}{*}{ Series } & \multirow{2}{*}{ Group } & \multicolumn{2}{|c|}{ MSE/ $A$ 5 time periods ahead } & \multicolumn{2}{|c|}{ MSE/ A 12 time periods ahead } \\
\hline & & (S)ARIMA & HW & (S)ARIMA & HW \\
\hline s1 & \multirow{7}{*}{1} & $6.8 \%$ & $6.8 \%$ & $27.0 \%$ & $22.4 \%$ \\
\hline s2 & & $59.3 \%$ & $79.6 \%$ & $73.0 \%$ & $74.2 \%$ \\
\hline s3 & & $33.0 \%$ & $41.8 \%$ & $469.9 \%$ & $111.5 \%$ \\
\hline s4 & & $35.1 \%$ & $28.2 \%$ & $43.8 \%$ & $48.9 \%$ \\
\hline s5 & & $5.1 \%$ & $20.2 \%$ & $12.9 \%$ & $34.5 \%$ \\
\hline s6 & & $2.6 \%$ & $5.1 \%$ & $22.0 \%$ & $7.9 \%$ \\
\hline s7 & & $434.4 \%$ & $3.9 \%$ & $10361.6 \%$ & $10675.5 \%$ \\
\hline s8 & \multirow{5}{*}{2} & $268.9 \%$ & $424.1 \%$ & $801.5 \%$ & $953.2 \%$ \\
\hline s9 & & $8.3 \%$ & $666.7 \%$ & $217.9 \%$ & $678.6 \%$ \\
\hline s10 & & $9.9 \%$ & $435.9 \%$ & $80.8 \%$ & $502.4 \%$ \\
\hline s11 & & $60.3 \%$ & $463.0 \%$ & $172.9 \%$ & $1424.8 \%$ \\
\hline s12 & & $529.8 \%$ & $286.0 \%$ & $439.7 \%$ & $1369.8 \%$ \\
\hline
\end{tabular}

Table 5: Comparison between (S)ARIMA and HW based on ME/ A.

\begin{tabular}{|c|c|c|c|c|c|}
\hline \multirow{2}{*}{ Series } & \multirow{2}{*}{ Group } & \multicolumn{2}{|c|}{ ME / $A 5$ time periods ahead } & \multicolumn{2}{|c|}{ ME/ $A 12$ time periods ahead } \\
\hline & & (S)ARIMA & HW & (S)ARIMA & HW \\
\hline s1 & \multirow{7}{*}{1} & $2.9 \%$ & $1.0 \%$ & $-1.7 \%$ & $0.4 \%$ \\
\hline s2 & & $-4.4 \%$ & $1.8 \%$ & $-5.0 \%$ & $-6.3 \%$ \\
\hline s3 & & $2.2 \%$ & $-6.6 \%$ & $6.7 \%$ & $18.7 \%$ \\
\hline s4 & & $0.8 \%$ & $0.4 \%$ & $1.3 \%$ & $9.5 \%$ \\
\hline s5 & & $-1.7 \%$ & $-1.4 \%$ & $-3.5 \%$ & $-5.1 \%$ \\
\hline s6 & & $-0.2 \%$ & $-1.8 \%$ & $-2.2 \%$ & $-2.3 \%$ \\
\hline s7 & & $-1.1 \%$ & $-0.2 \%$ & $5.1 \%$ & $5.2 \%$ \\
\hline s8 & \multirow{5}{*}{2} & $1.0 \%$ & $0.6 \%$ & $-32.0 \%$ & $-32.8 \%$ \\
\hline s9 & & $0.0 \%$ & $33.3 \%$ & $-25.0 \%$ & $64.3 \%$ \\
\hline s10 & & $0.8 \%$ & $-13.0 \%$ & $4.7 \%$ & $-5.1 \%$ \\
\hline s11 & & $-0.9 \%$ & $0.2 \%$ & $-1.4 \%$ & $24.6 \%$ \\
\hline s12 & & $-7.9 \%$ & $-0.4 \%$ & $-4.5 \%$ & $21.7 \%$ \\
\hline
\end{tabular}

the more useful the application of (S)ARIMA models is. In fact, even if the parsimony of the identified model is an issue dealt with extensively in literature, nowadays the availability of several commercial statistical software programs, which allow the user to test different (S)ARIMA models very quickly, let them become useful tools to be applied in different industrial contexts also for sporadic and irregular time series that present both seasonal and trend components.

Tables 4 and 5 show calculated values of MSE/ $A$ and ME/ $A$ indices for each time series, for forecasting of both 5 and 12 time periods ahead, divided into the aforementioned groups. Bold values are related with the best-performing approach.

MSE/ $A$ values obtained enforce considerations traced by analyzing MAD/ $A$ results. Otherwise, ME/ $A$ do not address an over- or underestimation. Sometimes the former behavior occurs, sometimes the latter. 


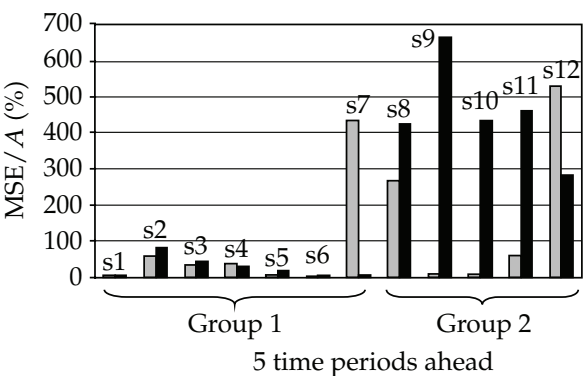

- (S)ARIMA

- HW

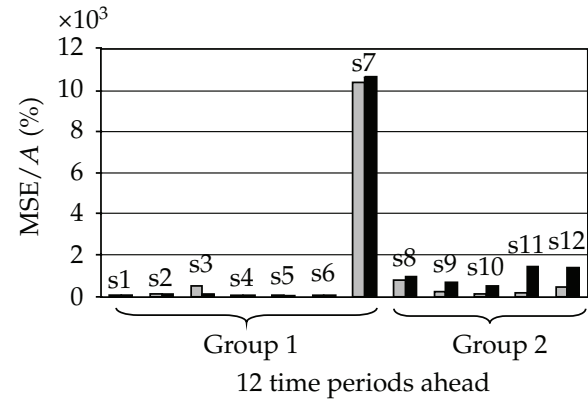

- (S)ARIMA

- HW

Figure 4: MSE/A values for each time series: forecasts on 5 and 12 time periods ahead.

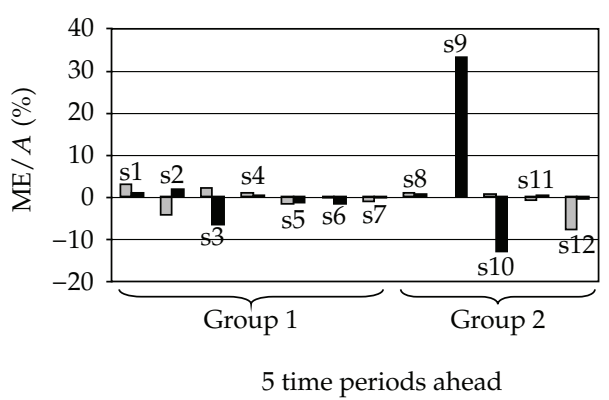

$\square$ (S)ARIMA

- HW

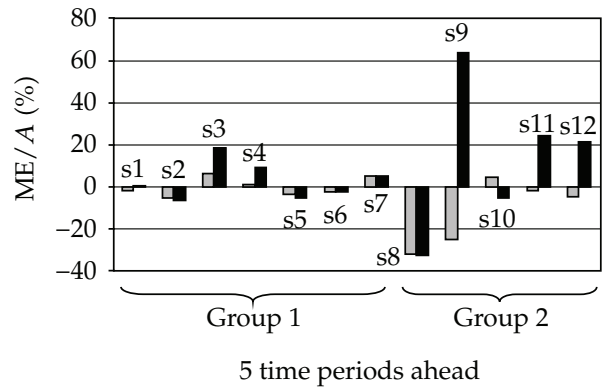

- (S)ARIMA

- HW

Figure 5: ME/ $A$ values for each time series: forecasts on 5 and 12 time periods ahead.

The results obtained corroborate the experimental analysis carried out by Bianchi et al. [28], which indicate that ARIMA is better than the best of additive or multiplicative Holt-Winters in more than three-fourths of the sample outcomes, and extend it in cases of sporadic and irregular time series. Specifically, this paper highlights that the performances of the (S)ARIMA models significantly improve when both seasonal and consistent trend components are present in time series.

\section{Conclusions}

The issue dealt with in the present paper is the comparison between the Holt-Winters method and the (S)ARIMA model for forecasting real life time series. In particular, the analyzed series present a high level of variability in terms of demand size and several null-demand time periods. Moreover, all of the time series reveal a clear seasonality while only several of these present a consistent trend component. On one hand, sporadic and irregular time series are extensively treated in literature, while on the other hand several authors compared the two methods above for seasonal and trendy time series. However, sporadic and irregular time series that present both trend and seasonal components are still neglected. Hence, evaluating the applicability of the (S)ARIMA and the Holt-Winters methods in forecasting sporadic 
demand time series with seasonality and trend components is the aim of the present paper in order to establish some useful guidelines for practitioners. The methodology applied consists of testing several (S)ARIMA models and then choosing the best model only in terms of forecasting performances, which is subsequently compared with the Holt-Winters method. In fact, statistical software programs also let the user test a robust and complex method like the (S)ARIMA very quickly and therefore the results from the comparison between the two methods can give a guidance for their applicability.

In particular, in the case of seasonality without a consistent trend component, the best (S)ARIMA model found and the Holt-Winters exponential smoothing model give similar results in terms of $\mathrm{MAD} / A$, but when also a consistent trend component is present, the performances of (S)ARIMA model are more appreciable. These results are enforced by the evaluation of MSE/A. Hence, when the sporadic demand data series structure becomes more complex because of the relevant presence of both seasonal and consistent trend components, the (S)ARIMA model, which is more adaptive than the Holt-Winters method, is also more effective.

This observation represents a useful decision-making guideline in plant management. In fact, several real contexts present these characteristics, such as startup productions, multiechelon supply chains or spare parts production, and selling, where demand forecasting constitutes an unforgettable prerequisite for an efficient production or selling management and could become a needful competitive leverage for companies.

As underlined in the introduction, when treating sporadic and irregular time series, two relevant issues refer to forecasting and inventory management. Further researches are addressed in the field of order and inventory management when sporadic demand data series with seasonality and consistent trend components are present. Furthermore, a comparative analysis on reachable performances when previously cited methodologies for sporadic demand forecasting with specifical hypothesis (i.e., demand distribution, time periods with zero demand distribution,...) are applied to forecasting intermittent series with seasonality and consistent trend components is addressed.

\section{Acknowledgments}

The authors wish to thank Eng. L. Biolchini for supporting this research by means of fruitful discussion and constructive criticism.

\section{References}

[1] J. D. Croston, "Forecasting and stock control for intermittent demands," Operational Research Quarterly, vol. 23, no. 3, pp. 289-303, 1972.

[2] A. Rao, "A comment on "Forecasting and stock control for intermittent demands"," Operational Research Quarterly, vol. 24, no. 4, pp. 639-640, 1973.

[3] F. R. Johnston and J. E. Boylan, "Forecasting for items with intermittent demand," Journal of the Operational Research Society, vol. 47, no. 1, pp. 113-121, 1996.

[4] F. R. Johnston and J. E. Boylan, "Forecasting intermittent demand: a comparative evaluation of Croston's method. Comment," International Journal of Forecasting, vol. 12, pp. 297-298, 1996.

[5] A. A. Syntetos and J. E. Boylan, "On the bias of intermittent demand estimates," International Journal of Production Economics, vol. 71, no. 1-3, pp. 457-466, 2001.

[6] A. A. Syntetos and J. E. Boylan, "The accuracy of intermittent demand estimates," International Journal of Forecasting, vol. 21, no. 2, pp. 303-314, 2005.

[7] A. Segerstedt, "Forecasting slow-moving items and ordinary items-a modification of Croston's idea," 
Working paper, Industrial logistics, Lulea University of Technology, 2000.

[8] E. Levén and A. Segerstedt, "Inventory control with a modified Croston procedure and Erlang distribution," International Journal of Production Economics, vol. 90, no. 3, pp. 361-367, 2004.

[9] T. R. Willemain, C. N. Smart, and H. F. Schwarz, "A new approach to forecasting intermittent demand for service parts inventories," International Journal of Forecasting, vol. 20, no. 3, pp. 375-387, 2004.

[10] R. S. Gutierrez, A. O. Solis, and S. Mukhopadhyay, "Lumpy demand forecasting using neural networks," International Journal of Production Economics, vol. 111, no. 2, pp. 409-420, 2008.

[11] T. R. Willemain, C. N. Smart, J. H. Shockor, and P. A. DeSautels, "Forecasting intermittent demand in manufacturing: a comparative evaluation of Croston's method," International Journal of Forecasting, vol. 10, no. 4, pp. 529-538, 1994.

[12] B. Sani and B. G. Kingsman, "Selecting the best periodic inventory control and demand forecasting methods for low demand items," Journal of the Operational Research Society, vol. 48, no. 7, pp. 700-713, 1997.

[13] L. W. G. Strijbosch, R. M. J. Heuts, and E. H. M. van der Schoot, "Combined forecast-inventory control procedure for spare parts," Journal of the Operational Research Society, vol. 51, no. 10, pp. 1184$1192,2000$.

[14] A. Regattieri, M. Gamberi, R. Gamberini, and R. Manzini, "Managing lumpy demand for aircraft spare parts," Journal of Air Transport Management, vol. 11, no. 6, pp. 426-431, 2005.

[15] A. A. Syntetos, "A note on managing lumpy demand for aircraft spare parts," Journal of Air Transport Management, vol. 13, no. 3, pp. 166-167, 2007.

[16] A. A. Syntetos, J. E. Boylan, and J. D. Croston, "On the categorization of demand patterns," Journal of the Operational Research Society, vol. 56, no. 5, pp. 495-503, 2005.

[17] L. Shenstone and R. J. Hyndman, "Stochastic models underlying Croston's method for intermittent demand forecasting," Journal of Forecasting, vol. 24, pp. 389-402, 2005.

[18] A. V. Kostenko and A. V. Hyndman, "A note on the categorization of demand patterns," Journal of the Operational Research Society, vol. 57, pp. 1256-1257, 2006.

[19] A. A. Syntetos, J. E. Boylan, and J. D. Croston, "Reply to Kostenko and Hyndman," Journal of the Operational Research Society, vol. 57, pp. 1257-1258, 2006.

[20] C. C. Holt, "Forecasting seasonals and trends by exponentially weighted moving averages," ONR Memorandum 52, Carnegie Institute of Technology, Washington, DC, USA, 1957.

[21] P. R. Winters, "Forecasting sales by exponentially weighted moving averages," Management Science, vol. 6, pp. 324-342, 1960.

[22] G. E. P. Box and G. M. Jenkins, Time Series Analysis: Forecasting and Control, Holden-Day Series in Time Series Analysis, Holden-Day, San Francisco, Calif, USA, Revised edition, 1976.

[23] J. Jarrett, Business Forecasting Methods, Blackwell, Oxford, UK, 1991.

[24] B. L. Bowerman and R. T. O'Connell, Forecasting and Time Series: An Applied Approach, Duxbury Press, Belmont, Calif, USA, 1993.

[25] P. Newbold and C. W. J. Granger, "Experience with forecasting univariate time series and the combination of forecasts," Journal of the Royal Statistical Society. Series A, vol. 137, pp. 131-165, 1974.

[26] L. Poulos, A. Kvanli, and R. Pavur, "A comparison of the accuracy of the Box-Jenkins method with that of automated forecasting methods," International Journal of Forecasting, vol. 3, no. 2, pp. 261-267, 1987.

[27] C. Chen, "Robustness properties of some forecasting methods for seasonal time series: a Monte Carlo study," International Journal of Forecasting, vol. 13, no. 2, pp. 269-280, 1997.

[28] L. Bianchi, J. Jarrett, and R. C. Hanumara, "Improving forecasting for telemarketing centers by ARIMA modeling with intervention," International Journal of Forecasting, vol. 14, no. 4, pp. 497-504, 1998.

[29] H. Hassani, S. Heravi, and A. Zhigljavsky, “Forecasting European Industrial Production with Singular Spectrum Analysis," International Journal of Forecasting. In press.

[30] F. Canova and B. E. Hansen, "Are seasonal patterns constant over time? A test for seasonal stability," Journal of Business and Economic Statistics, vol. 13, pp. 237-252, 1995.

[31] H. Akaike, "A new look at the statistical model identification," IEEE Transactions on Automatic Control, vol. 19, no. 6, pp. 716-723, 1974.

[32] G. E. Schwarz, "Estimating the dimension of a model," The Annals of Statistics, vol. 6, no. 2, pp. 461464, 1978.

[33] S. Makridakis, "Accuracy measures: theoretical and practical concerns," International Journal of Forecasting, vol. 9, no. 4, pp. 527-529, 1993. 


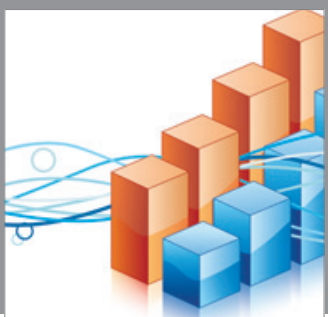

Advances in

Operations Research

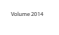

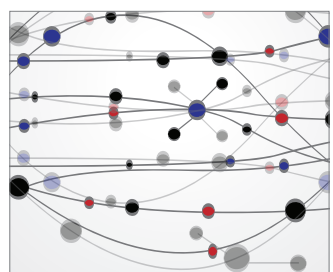

\section{The Scientific} World Journal
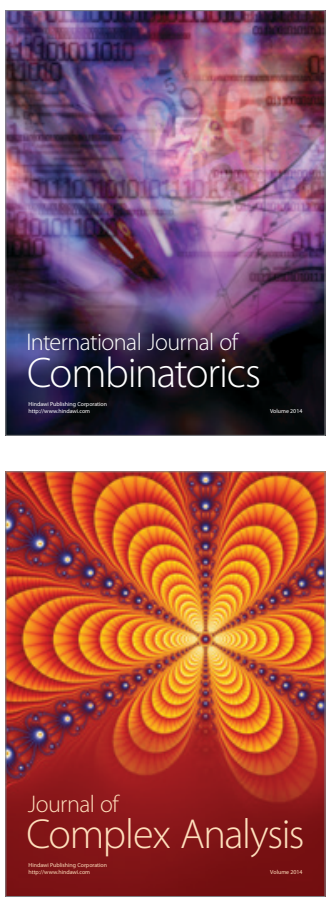

International Journal of

Mathematics and

Mathematical

Sciences
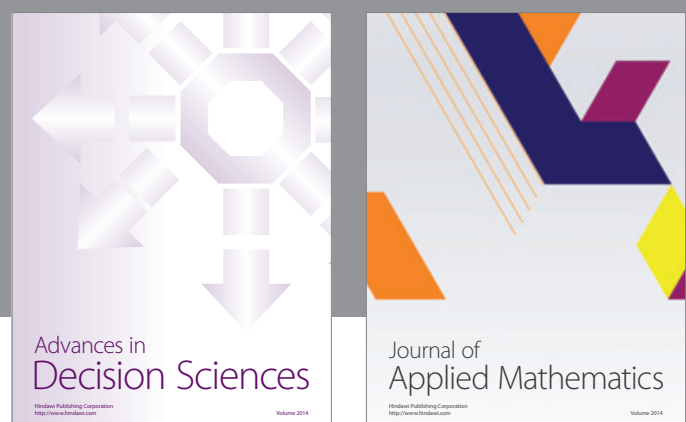

Journal of

Applied Mathematics
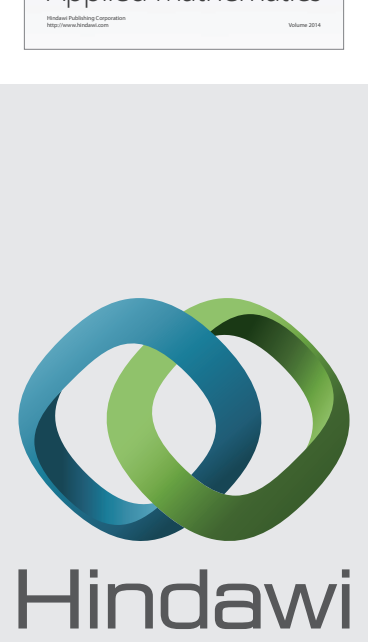

Submit your manuscripts at http://www.hindawi.com
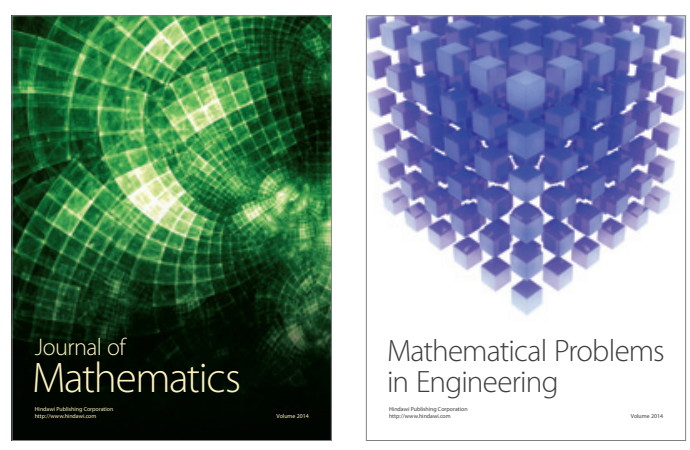

Mathematical Problems in Engineering
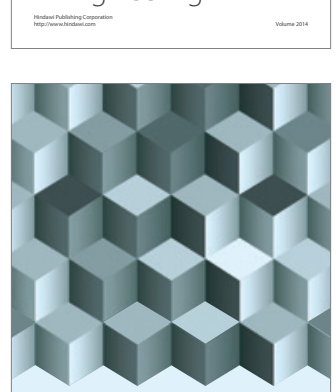

Journal of

Function Spaces
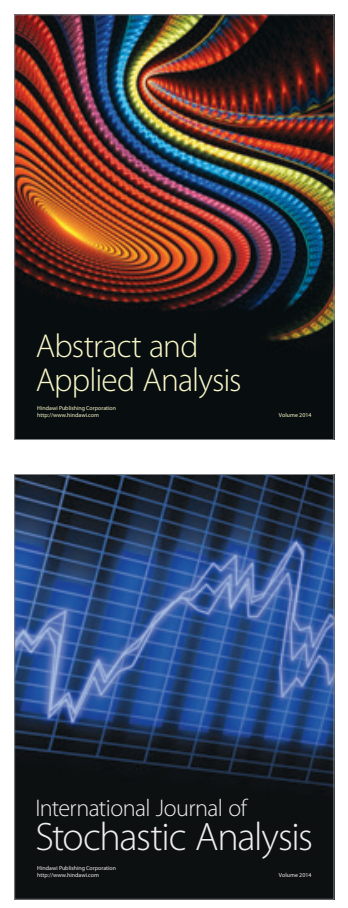

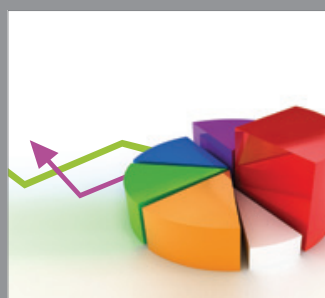

ournal of

Probability and Statistics

Promensencen
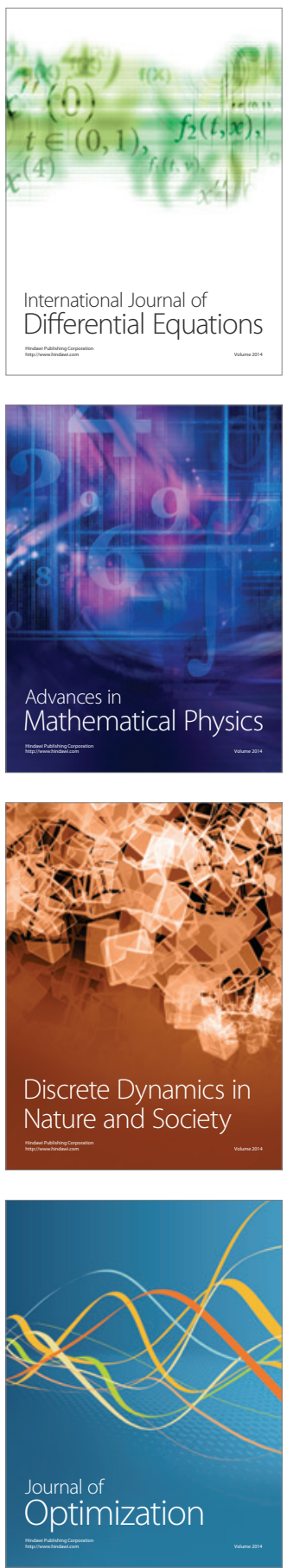